\title{
Phytoprotection
}

\section{Les nématodes, ces anguillules qui font suer les plantes... par la racine}

\section{Guy Bélair}

Volume 86, numéro 1, avril 2005

URI : https://id.erudit.org/iderudit/011717ar

DOI : https://doi.org/10.7202/011717ar

Aller au sommaire du numéro

\section{Éditeur(s)}

Société de protection des plantes du Québec (SPPQ)

\section{ISSN}

0031-9511 (imprimé)

1710-1603 (numérique)

Découvrir la revue

\section{Citer cet article}

Bélair, G. (2005). Les nématodes, ces anguillules qui font suer les plantes... par la racine. Phytoprotection, 86(1), 65-69. https://doi.org/10.7202/011717ar

\section{Résumé de l'article}

Les nématodes phytoparasites ou anguillules sont d'importants ravageurs des plantes en agriculture. À l'échelle de la planète, les nématodes occasionnent plus de 100 milliards de dollars en perte de production annuellement. Ces petits vers microscopiques, tous munis d'un stylet creux, sont transparents et mesurent de 300 à $1500 \mu \mathrm{m}$ de longueur et de 15 à $35 \mu \mathrm{m}$ de diamètre. Leur petit diamètre ne permet pas de les voir à l'oeil nu, mais ils sont facilement observables sous la loupe binoculaire. Au Canada, on rapporte un total de 19 genres et 37 espèces de nématodes associés à une maladie des plantes. La majorité, soit 16 genres et 31 espèces, parasitent le système racinaire des plantes. Ils peuvent occasionner des dommages à la plante variant de négligeables jusqu'à une perte totale. La sévérité des dommages occasionnés aux plantes est reliée à plusieurs facteurs comme la combinaison plante-nématode et aussi aux facteurs environnementaux comme les précipitations, le type de sol et les pratiques culturales. Dans leur processus de nutrition, ils ponctionnent à l'aide de leur stylet le système racinaire de la plante, ce qui diminue la capacité d'absorption de l'eau et des éléments nutritifs dans le sol. Des symptômes typiques d'un dommage causé par les nématodes sont une réduction du système racinaire, une distorsion de la structure racinaire ou l'augmentation du diamètre des racines. Parmi les ravageurs prépondérants, mentionnons les espèces endoparasites suivantes : le nématode cécidogène du nord Meloidogynehapla Chitwood, le nématode des lésions Pratylenchuspenetrans (Cobb) Filipjev \& Schuurmans-Stekhoven, le nématode des tiges et des bulbes Ditylenchus dipsaci (Kühn) Filipjev, le nématode dague Xiphinema rivesi Dalmasso, et le nématode à kystes du soja Heterodera glycines Ichinohe. Même s'ils sont répertoriés au Canada, certains nématodes comme le nématode doré de la pomme de terre Globodera rostochiensis (Wollenweb.) Behrens et G.pallida (Stone) Behrens sont des organismes de quarantaine et doivent être interceptés par l'Agence canadienne d'inspection des aliments afin de limiter leur distribution sur le territoire. $\mathrm{Au}$ Canada, le contrôle des nématodes s'effectue avec des méthodes culturales (ex. rotation, résistance) et chimiques.
Tous droits réservés @ La société de protection des plantes du Québec, 2005
Ce document est protégé par la loi sur le droit d'auteur. L'utilisation des services d'Érudit (y compris la reproduction) est assujettie à sa politique d'utilisation que vous pouvez consulter en ligne.

https://apropos.erudit.org/fr/usagers/politique-dutilisation/ 


\title{
Les nématodes, ces anguillules qui font suer les plantes... par la racine
}

\author{
Guy Bélair ${ }^{1}$
}

\section{PHYTOPROTECTION 86 : 65-69}

Les nématodes phytoparasites ou anguillules sont d'importants ravageurs des plantes en agriculture. À l'échelle de la planète, les nématodes occasionnent plus de 100 milliards de dollars en perte de production annuellement. Ces petits vers microscopiques, tous munis d'un stylet creux, sont transparents et mesurent de 300 à $1500 \mu \mathrm{m}$ de longueur et de 15 à $35 \mu \mathrm{m}$ de diamètre. Leur petit diamètre ne permet pas de les voir à l'œil nu, mais ils sont facilement observables sous la loupe binoculaire. Au Canada, on rapporte un total de 19 genres et 37 espèces de nématodes associés à une maladie des plantes. La majorité, soit 16 genres et 31 espèces, parasitent le système racinaire des plantes. Ils peuvent occasionner des dommages à la plante variant de négligeables jusqu'à une perte totale. La sévérité des dommages occasionnés aux plantes est reliée à plusieurs facteurs comme la combinaison plante-nématode et aussi aux facteurs environnementaux comme les précipitations, le type de sol et les pratiques culturales. Dans leur processus de nutrition, ils ponctionnent à l'aide de leur stylet le système racinaire de la plante, ce qui diminue la capacité d'absorption de l'eau et des éléments nutritifs dans le sol. Des symptômes typiques d'un dommage causé par les nématodes sont une réduction du système racinaire, une distorsion de la structure racinaire ou l'augmentation du diamètre des racines. Parmi les ravageurs prépondérants, mentionnons les espèces endoparasites suivantes : le nématode cécidogène du nord Meloidogyne hapla, le nématode des lésions Pratylenchus penetrans, le nématode des tiges et des bulbes Ditylenchus dipsaci, le nématode dague Xiphinema rivesi, et le nématode à kystes du soja Heterodera glycines. Même s'ils sont répertoriés au Canada, certains nématodes comme le nématode doré de la pomme de terre Globodera rostochiensis et G. pallida sont des organismes de quarantaine et doivent être interceptés par l'Agence canadienne d'inspection des aliments afin de limiter leur distribution sur le territoire. Au Canada, le contrôle des nématodes s'effectue avec des méthodes culturales (ex. rotation, résistance) et chimiques.

Mots clés: Globodera, Heterodera, Meloidogyne, méthodes de contrôle, Pratylenchus, quarantaine, Xiphinema.

\section{[Nematodes, these roundworms that harm plants... by their roots]}

Plant-parasitic nematodes or round worms are major pests in agriculture. Worldwide, nematodes cause more than $\$ 100$ billion in losses annually. These small worm-like organisms, each equipped with a hollow stylet, are transparent and measure from 300 to $1500 \mu \mathrm{m}$ in length and 15 to $35 \mu \mathrm{m}$ in diameter. Their small diameter does not allow us to see them with the naked eye but they are easily observed under a stereomicroscope. In Canada, there are 19 genera and 37 species of plant-parasitic nematodes associated with a plant disease. The majority, 16 genera and 31 species, parasitize the root system. They can cause a wide range of plant damage, from somewhat negligible to total crop loss. The severity of damage caused to the plants is related to various factors including the plant-nematode combination, environmental factors such water precipitation, soil type, and also cultural practices. When feeding, they puncture the root with their hollow stylet and thus reduce the capacity of water and nutritious elements uptake by the root system. The typical symptoms of nematode feeding are the reduction in root system, a distortion of the root structure and an increase in root diameter. Among the most prevalent pests, we must include the following endoparasitic species: the northern root-knot nematode Meloidogyne hapla, the root-lesion nematode Pratylenchus penetrans, the stem and bulb nematode Ditylenchus dipsaci, the dagger nematode Xiphinema rivesi, and the soybean cyst nematode Heterodera glycines. Even though they are found in Canada, nematodes such as the potato cyst nematode Globodera rostochiensis and G. pallida are quarantine organisms and must be intercepted by the Canadian Food Inspection Agency

1. Centre de recherche et de développement en horticulture, Agriculture et Agroalimentaire Canada, Saint-Jean-sur-Richelieu (Québec), Canada J3B 3E6; courriel : belairg@agr.qc.ca 
to prevent them from spreading over the territory. In Canada, the management of plant-parasitic nematodes is ensured by cultural methods (e.g., crop rotation, resistance) and chemicals.

Keywords: Control methods, Globodera, Heterodera, Meloigogyne, Pratylenchus, quarantine, Xiphinema.

\section{INTRODUCTION}

Dans le règne animal, les nématodes sont tous regroupés sous le Phylum Nematoda faisant partie du Superphylum Ecdysozoa, du Grec ecdysis (mue) et zoon (animal). Ce dernier comprend les arthropodes (insectes, araignées et crustacées), les rotifères, les nématomorphes, les tartigrades et les nématodes. Ils possèdent tous une cuticule et doivent muer pour croître. Contrairement aux invertébrés qui sont de proches parents, les nématodes ont un corps mou et les recherches jusqu'à maintenant $n^{\prime}$ ont pas permis de retrouver beaucoup de spécimens fossilisés, ce qui limite nos connaissances sur la forme des espèces ancestrales. Le premier fossile de nématode a été trouvé dans l'ambre (sève d'arbre fossilisée) associé à un insecte hôte, ce qui remonte à environ 125 millions d'années (Nickle 1984). Il est clair que les nématodes comme groupe sont apparus beaucoup plus tôt mais le processus de fossilisation ne permet pas d'associer ce type d'organisme à des dates antérieures.

La majorité des nématodes sont dits "libres" et se nourrissent essentiellement de bactéries, de champignons, de protozoaires et d'autres nématodes. C'est donc une minorité des nématodes qui parasite les animaux et les plantes. La première observation suggérant un nématode parasite de plantes sur racine de soja a été notée en Chine antique, il y a environ 2000 ans. La première description d'un nématode parasite de plantes, soit Anguina tritici (Steinbuch) Chitwood dans des grains de blé, a été effectuée par le révérend Tuberville Needham en I'an 1743 (Nickle 1984).

La nématologie est vraiment devenue une discipline scientifique au $19^{\mathrm{e}}$ siècle lorsque Berkeley publie en 1855 une description du nématode cécidogène Meloidogyne sur le concombre et que Schacht, en 1859, décrit le nématode à kyste Heterodera schachtii Schmidt sur la betterave sucrière. Au cours des années 1940, l'avènement de la fumigation du sol a permis de démontrer dans plusieurs systèmes de production agricole que les nématodes étaient d'importants ravageurs des cultures (Nickle 1984).

\section{Les nématodes phytoparasites au Canada}

Au Canada, on répertorie 72 plantes hôtes sur 550 plantes inscrites avec une ou plus d'une maladie causée par les nématodes, soit $13 \%$, avec la répartition suivante : 32 dans les céréales ou plantes fourragères, 18 dans les plantes ornementales, 15 dans les cultures fruitières et maraîchères et 7 dans les arbres forestiers (SPPQ 2003). On rapporte un total de 19 genres et 37 espèces de nématodes associés avec une maladie des plantes. La majorité, soit 16 genres et 31 espèces, parasite le système racinaire des plantes et occasionne des dommages variant de négligeables allant à une perte totale. Parmi les ravageurs prépondérants, mentionnons les espèces endoparasites suivantes : le nématode cécidogène du nord Meloidogyne hapla Chitwood, le nématode des lésions Pratylenchus penetrans (Cobb) Filipjev \& Schuurmans-Stekhoven, le nématode des tiges et des bulbes Ditylenchus dipsaci (Kühn) Filipjev, le nématode dague Xiphinema rivesi Dalmasso, et le nématode à kystes du soja Heterodera glycines Ichinohe (Rousson 2005).

Comparativement à nos voisins du sud, soit les États-Unis, le Canada héberge beaucoup moins de nématodes pathogènes des plantes. Plusieurs facteurs semblent contribuer à limiter leur nombre sur notre territoire, comme le climat nordique, une production agricole moins intensive ainsi que le contrôle des introductions par l'Agence canadienne d'inspection des aliments (Rousson 2005).

En 1987, on a estimé les pertes annuelles causées par les nématodes à l'échelle mondiale à plus de 100 milliards en dollars canadiens. Au Canada et aux États-Unis, ces pertes sont estimées respectivement à 1 et 7 milliards en dollars canadiens.

\section{Des généralités sur les nématodes phytoparasites}

Le processus est le même pour tous les nématodes phytoparasites. Ils ponctionnent les cellules à l'aide de leur stylet creux, sécrètent des protéines et d'autres métabolites pour les assister dans leur parasitisme et, ultimement, aspirent le contenu cellulaire. Les racines endommagées sont diminuées dans leur capacité d'absorption de l'eau et des éléments nutritifs dans le sol. Les blessures causées aux cellules des racines fournissent une opportunité pour les autres pathogènes (bactéries, champignons, virus) de s'introduire dans les racines. Les symptômes typiques causés par les nématodes aux racines sont une réduction du système racinaire, une distorsion de la structure racinaire ou une augmentation du diamètre des racines. La sévérité des dommages occasionnés est reliée surtout à la combinaison plante-nématode, à des facteurs environnementaux comme les précipitations (humidité du sol), le type de sol et les pratiques culturales (ex. cultivar résistant).

Le cycle de développement de la plupart des nématodes phytoparasites est en général très similaire d'une espèce à une autre. Les oeufs éclosent en larves lesquelles ont une apparence et une structure habituellement semblables aux adultes. Les larves grossissent et chaque stade larvaire se termine par une mue. Tous les nématodes possèdent quatre stades larvaires, avec le premier stade larvaire généralement dans l'œuf. La différenciation sexuelle s'effectue après la quatrième mue. Les femelles produisent des œufs fertiles par reproduction sexuée, parthénogenèse ou hermaphrodisme. Chez certaines 
espèces, les mâles sont absents. Pour plusieurs espèces, le cycle complet s'effectue en 3-4 semaines en fonction de la température et de la plante. Le deuxième stade larvaire qui est libre dans le sol possède une courte réserve d'énergie et doit se nourrir sur une plante-hôte, sinon c'est la mort de tous les individus de cette espèce dans les quelques mois suivants. En absence d'un hôte favorable, les stades larvaires de certaines espèces peuvent se déshydrater et demeurer en quiescence ou bien les œufs restent en dormance dans le sol pendant des années.

\section{Un peu d'écologie}

Les nématodes parasites des racines passent une grande proportion du temps dans le sol. Pour les espèces ectoparasites, tous les stades se retrouvent dans le sol. Dans le cas des endoparasites sédentaires, les oeufs, les larves de deuxième stade $\left(L_{2}\right)$ et les mâles sont dans le sol alors que les $L_{3}, L_{4}$ et les femelles sont dans la racine. En général, les œufs éclosent librement dans l'eau sans stimulus. Toutefois, pour certaines espèces, les exsudats racinaires de la plante hôte sont nécessaires pour stimuler l'éclosion des œufs, comme par exemple chez le nématode doré de la pomme de terre. Les nématodes sont plus abondants dans la couche $0-20 \mathrm{~cm}$, ce qui correspond grosso modo à la zone des racines. La température, I'humidité et la texture du sol jouent un rôle important sur la survie et la mobilité des nématodes. Par lui-même, un nématode se déplace sur de très courtes distances, soit quelques dizaines de centimètres par année. La dispersion sur de grandes distances est assurée par le sol (les oiseaux, les mammifères, les humains), les plants ou semences contaminés, le vent, l'eau et l'équipement agricole. Dans le champ, leur distribution est généralement hétérogène et morcelée.

En ce qui concerne les niches, on peut regrouper tous les nématodes parasites des racines en deux grandes catégories, soit les ectoparasites et les endoparasites. Chez les endoparasites, on trouve deux sous-groupes, les migrateurs et les sédentaires. Les ectoparasites sont toujours dans le sol et utilisent leur stylet pour se nourrir des cellules de la racine. Cette stratégie permet aux nématodes de se déplacer librement et de brouter sur plusieurs racines de plantes différentes bien que leur mobilité accrue les rende plus vulnérables face aux fluctuations environnementales et à la prédation. Les ectoparasites se nourrissent typiquement de cellules près de la surface (ex. Helicotylenchus, Paratylenchus), mais ils peuvent aussi être munis d'un stylet extrêmement long leur permettant de se nourrir à l'intérieur de la racine sur des cellules riches en éléments nutritifs (ex. Xiphinema). Au Canada, la plupart des ectoparasites ne sont pas des ravageurs des cultures, comme par exemple les Helicotylenchus spp., les Crinomema spp. et les Paratylenchus spp.

Les nématodes phytoparasites les plus dommageables au monde appartiennent au type endoparasite sédentaire. Les deux principaux genres de ce groupe sont les nématodes à kystes (Heterodera et Globodera) et les nématodes à galles (Meloidogyne). Chez ces nématodes, la larve de deuxième stade pénètre derrière l'apex de la racine et migre vers les cellules vasculaires en développement. Au cours des premiers stades de développement, les nématodes sont complètement enchâssés dans la racine mais plus tard font saillie sur la racine. La larve de deuxième stade qui est maintenant devenue sédentaire injecte des sécrétions dans les cellules périphériques pour stimuler la formation de cellules nourricières sur lesquelles le nématode se nourrit de manière non destructive tout au long de son développement. Chez les Heterodera, les œufs demeurent à l'intérieur de la femelle, laquelle se dilate en forme de poire. Lorsque la femelle meurt, sa cuticule s'épaissit, se durcit et se transforme en un kyste. Chez les Meloidogyne, les femelles pondent leurs œufs à l'intérieur ou à l'extérieur de la racine dans une gangue gélatineuse.

Les nématodes endoparasites migrateurs passent une bonne majorité de leur temps à se déplacer dans les tissus racinaires afin de se nourrir des cellules qu'ils détruisent. En aspirant le contenu cellulaire et en se déplaçant d'une cellule à l'autre, ils peuvent occasionner des nécroses massives dans les tissus attaqués. Le nématode des lésions Pratylenchus, le nématode des tiges et des bulbes Ditylenchus et le nématode lance Hoplolaimus sont tous des représentants de ce type.

\section{Quelques exemples de nématodes des racines sous haute surveillance}

1. Le nématode dague Xiphinema spp.

Les nématodes appartenant au genre Xiphinema sont tous des ectoparasites et d'importants vecteurs de virus. Tous les stades se nourrissent sur I'apex racinaire de la plante. Muni d'un très long stylet, il le pénètre loin dans l'apex et occasionne une hypertrophie des cellules et un épaississement des parois endommagées. Avec une longévité de 3 à 5 ans, ces ectoparasites n'ont qu'une seule génération par année et se maintiennent presque exclusivement sur les cultures pérennes. Sur les racines affectées, ils occasionnent un rabougrissement et une nodulation. En absence de particules virales, le seuil de nuisibilité est fixé autour de 500 nématodes par $100 \mathrm{~cm}^{3}$ de sol. Par contre, s'ils transportent avec eux des particules virales, le seuil est réduit à quelques individus seulement par $100 \mathrm{~cm}^{3}$ de sol puisqu'un seul nématode sera suffisant pour transmettre le virus. Les nématodes du genre Xiphinema sont tous vecteurs des NEPO virus (NEmatode POlyhedral particles) ce qui comprend des virus comme les virus de la tache annulaire du tabac (TRSV) et de la tomate (TmRSV). Au Canada, ce genre de nématode constitue donc une menace réelle pour toute la production des petits fruits, des arbres fruitiers ainsi que des arbres forestiers.

La quarantaine est un moyen efficace pour empêcher l'importation de vignes infectées au pays. Actuellement, il existe peu ou pas de matériel végétal possédant une résistance à ce genre de nématode. Après la transplantation, la désinfection du sol à l'aide de la fumigation avec un nématicide est une pratique courante dans les cultures fruitières comme la vigne afin de réduire les risques d'infection.

2. Le nématode doré et le nématode à kystes pâles Globodera rostochiensis et $\mathrm{G}$. pallida

Le nématode doré et le nématode à kystes pâles Globodera rostochiensis (Wollenweb.) Behrens et 
G. pallida (Stone) Behrens sont des nématodes de quarantaine qui constituent des menaces importantes et réelles pour le marché de la pomme de terre au Canada, notamment pour la production de semence. Globodera rostochiensis et G. pallida sont présents dans la province de Terre-Neuve et G. rostochiensis sur l'île de Victoria en ColombieBritannique. Les deux espèces de nématodes à kystes de la pomme de terre sont des parasites obligatoires de certains membres de la famille des solanacées. La pomme de terre (Solanum spp.) est I'hôte principal, suivi de la tomate (Lycopersicon esculentum Mill.) et de I'aubergine (S. melongena L.). Au total, quelques 90 espèces du genre Solanum sont reconnues comme étant des hôtes de ces ravageurs. À maturité, les femelles de $G$. rostochiensis tournent du blanc au jaune avant de constituer un kyste foncé. Celles de G. pallida n'ont pas de phase dorée et leur couleur varie de blanc à crème, puis elles foncent progressivement. Un kyste peut contenir jusqu'à 500 oeufs et est en mesure de persister dans le sol pendant plus de 20 ans.

Le travail de quarantaine effectué par l'Agence canadienne d'inspection des aliments permet de bloquer les introductions en provenance des nombreux pays importateurs et aussi de contenir ces mêmes espèces sur notre propre territoire. Plusieurs variétés de pomme de terre résistantes ont été développées en Europe et sont disponibles. À cause de la très longue persistance des kystes dans le sol, le retour en pomme de terre et autres plantes hôtes dans les champs infestés peut se faire seulement après 5 à 10 ans de rotation. La fumigation du sol est une méthode de lutte efficace mais très onéreuse. En lutte biologique, des champignons parasites des œufs comme les Verticillium chlamydosporium et Paecilomyces lilacinus ont fait l'objet de recherche et développement. Ce dernier est présentement commercialisé $\left(\right.$ Bioact $^{\circledR}$, Bioact Corporation Pty Ltd., Sydney, Australie) contre les nématodes à kystes et les nématodes à galles.

3. Le nématode à kystes du soja Heterodera glycines Même si on le retrouve au Canada, le nématode à kystes du soja Heterodera glycines demeure un nématode de quarantaine. Il a été signalé pour la première fois au Canada en 1987 (sud-ouest de I'Ontario). Les nouveaux foyers d'infestation peuvent être occasionnés par le transport de kystes de champs infestés à des champs non infestés, par la machinerie souillée de sol, par les oiseaux ou le vent. Heureusement le soja Glycine max L. Merro est la seule culture d'importance économique qui peut être gravement attaquée. On reconnaît actuellement au moins 16 races du nématode à kystes du soja morphologiquement semblables, mais physiologiquement différentes. En Ontario, la Race 3 est dite active.

II est nécessaire de maintenir ce nématode comme organisme de quarantaine afin de limiter sa dispersion au pays et possiblement de réduire son impact dans les régions déjà infestées du sud de l'Ontario. À moyen et à long terme, il est probable que ce parasite va continuer à se propager sur le territoire canadien. Des cultivars résistants sont disponibles, mais il est suggéré d'effectuer une rotation des variétés pour éviter la perte de résistance. Dans un programme de rotation de 4 ans, il est recommandé de faire deux années d'une culture non hôte, suivies d'une première année de soja avec une variété résistante et par la suite une deuxième année avec une variété sensible (Dufour et al. 2003). La fumigation du sol est un moyen efficace mais peu rentable économiquement contre ce ravageur.

4. Le nématode des lésions Pratylenchus penetrans Contrairement aux nématodes décrits précédemment, le nématode des lésions Pratylenchus penetrans n'est pas un organisme de quarantaine et est une espèce indigène. C'est un endoparasite migrateur très répandu dans les sols sableux, soit les champs de pomme de terre, de tabac, de fraise et de framboise. En effet, $P$. penetrans est de loin l'espèce la plus importante économiquement au Canada, dans le nord-est des États-Unis et dans certaines parties d'Europe. En Ontario et au Québec, le seuil de nuisibilité de cette espèce a été établi à 100 nématodes par $100 \mathrm{~cm}^{3}$ de sol ou 1000 par $\mathrm{kg}$ de sol (Bélair et al. 2005; Potter et Olthof 1974). Dans la culture de la pomme de terre, des pertes de rendement de 10 à $40 \%$ peuvent être occasionnées par ce nématode. Très polyphage, il peut se nourrir sur une multitude de plantes d'importance agricole, incluant les céréales et les plantes fourragères, ainsi que sur la plupart des mauvaises herbes (Bélair et al. 2002). Des effets additifs et synergiques avec d'autres organismes pathogènes du sol comme les Verticillium albo-atrum Reinke \& Berthier et $V$. dahliae Kleb. sont observés notamment dans la famille des Solanacées. Au Canada et aux États-Unis, le dépérissement hâtif de la pomme de terre occasionne d'importantes pertes de rendement dans cette culture, un exemple étant la synergie entre la verticilliose et $P$. penetrans.

Dans les sols légers à pomme de terre, le seigle est cultivé traditionnellement comme culture de rotation puisqu'elle est bien adaptée à ces sols secs et peu fertiles. Toutefois, cette culture permet de multiplier les populations du $P$. penetrans avec des densités pouvant facilement atteindre le seuil de nuisibilité. Des essais en serre et en champ ont permis de démontrer le potentiel du millet perlé Pennisetum glaucum comme culture de rotation qui permet de réduire les populations du nématode et d'augmenter les rendements de pomme de terre l'année subséquente (Bélair et al. 2005). Dans un essai de cultures de rotation dans la région de Québec, le millet perlé semble également prometteur pour réduire les pertes et dommages causés par un autre complexe nématodeverticilliose dans la culture de la fraise (Coulombe et al. 2003).

\section{Limiter la dispersion des nématodes à la ferme} Comme première ligne de front dans la lutte contre les nématodes, la prévention demeure une méthode importante et efficace d'intervention. Plusieurs mesures contribuent à limiter la dispersion faite par les producteurs sur leur propre ferme ou ailleurs : 1/ I'utilisation de matériel végétal certifié (semence et plants); 2/ l'emploi de milieu de croissance sans sol pour les cultures en serre; 3/ un nettoyage de la machinerie agricole souillée de sol avant d'entrer dans un autre champ; 4/ un bon emploi de l'eau d'irrigation, en récupérant les excès d'eau dans un bassin de sédimentation et en pompant l'eau près de 
la surface de l'étang; 5/ le contrôle du mouvement des animaux d'un champ infesté à un autre non infesté; 6/ I'élimination des mauvaises herbes comme plante-hôte; 7/ le compostage des fumiers avant de les épandre au champ (Dufour et al. 2003).

\section{Méthodes alternatives de lutte}

La solarisation et I'inondation estivale sont deux méthodes de lutte qui ont une efficacité très limitée sous nos conditions climatiques tempérées. Toutefois, l'inondation tard à I'automne augmente la formation de glace dans le sol et diminue la survie des nématodes lors de nos hivers rigoureux. Les amendements de sol avec des composts, des fumiers bien compostés et des engrais verts stimulent la flore microbienne des sols et ont un effet de répression sur les populations de nématodes phytoparasites. Même si le mécanisme d'action n'est pas encore élucidé, les applications au sol de nématodes entomopathogènes du genre Steinernema réduisent les populations de nématodes phytoparasites. En lutte biologique, plusieurs microorganismes du sol ont fait l'objet de recherche contre les nématodes. Quatre produits, soit deux champignons, Paecilomyces lilacinus (BioAct ${ }^{\mathrm{TM}}$ ) et Myrothecium verrucaria (DiTera ${ }^{\mathrm{TM}}$ ), et deux bactéries, Burkholderia cepacia (Deny ${ }^{\mathrm{TM}}$ ) et Bacillus chitinosporus (Activate ${ }^{T M}$ ), sont présentement disponibles dans divers pays d'Europe et aux États-Unis.

\section{CONCLUSION}

Au Canada, les nématodes phytoparasites des racines occasionnent présentement et continueront d'infliger d'importantes pertes et dommages à plusieurs cultures horticoles, céréalières et commerciales. Parce que les nématodes ne sont pas visibles à l'œil nu et que les symptômes sur la partie aérienne des plantes peuvent être confondus avec plusieurs autres désordres du système racinaire, les nématodes sont le plus souvent oubliés par les producteurs et agronomes au moment de faire un diagnostic au champ. En recherche et développement, il y a eu depuis déjà une dizaine d'années une réduction majeure du personnel et des ressources dans plusieurs disciplines appliquées, y compris la nématologie. Avec l'intensification de la production, le réchauffement de la planète et la réduction de la biodiversité, il est très probable que les problèmes phytosanitaires en agriculture vont évoluer et possiblement se complexifier dans un avenir plus ou moins rapproché. Afin de contrer les problèmes futurs, une sensibilisation et une formation des producteurs agricoles s'imposent à l'échelle du pays afin qu'ils emploient de bonnes pratiques phytosanitaires à la ferme. À la frontière, une vigilance accrue des autorités gouvernementales en matière de quarantaine et d'inspection des végétaux introduits au pays est essentielle dans le but de protéger l'avenir de la production canadienne.

Dans le passé, la lutte contre les nématodes phytoparasites a joué un rôle de premier plan dans le progrès de la production agricole mondiale. Toutefois, I'emploi de certains produits chimiques, comme le 1,2-dibromo-3-chloropropane (DBCP), a contaminé le sol et les eaux souterraines et a conduit à leur bannissement du marché à l'échelle de la planète. Les recherches actuelles s'orientent davantage vers une lutte intégrée et durable des populations de nématodes par la manipulation génétique des plantes-hôtes, la conception de programmes de rotation, le développement de produits chimiques d'origine botanique et la lutte biologique. II y a donc présentement un effort marqué pour passer de la lutte chimique et l'emploi des nématicides aux méthodes dites alternatives. Parce que les approches alternatives sont davantage spécifiques au ravageur et au système de production, des liens entre la recherche appliquée et la recherche de base sur la biologie des nématodes doivent être supportés et maintenus pour promouvoir le développement de nouvelles technologies.

\section{REMERCIEMENTS}

Je tiens à remercier Yvon Fournier, Nathalie Dauphinais, Jean Coulombe, Marc F. Clément et Om. P. Dangi pour leur précieux support et leur collaboration.

\section{RÉFÉRENCES}

Bélair, G., Y. Fournier, N. Dauphinais et O.P. Dangi. 2002. Reproduction of Pratylenchus penetrans on various rotation crops in Quebec. Phytoprotection 83 : 111-114.

Bélair, G., N. Dauphinais, Y. Fournier, O.P. Dangi et M.F Clément. 2005. Effect of forage and pearl millet on Pratylenchus penetrans and potato yields in Quebec. J. Nematol. 37 : 78-82.

Coulombe, J., P. Lamy, G. Bélair et P.O. Thibodeau. 2003. Méthodes de contrôle de la flétrissure verticillienne et des nématodes dans la culture du fraisier et la pomme de terre. Ministère de l'Agriculture, des Pêcheries et de I'Alimentation du Québec. Conseil de développement en agriculture du Québec - Club Agroenvironnemental de la Rive-Nord. $47 \mathrm{pp}$.

Dufour, R., M. Guerena et R. Earles. 2003. Alternative nematode control. Pest Management Technical Note. ATTRA National Sustainable Agriculture Information Service. http://www.attra.org/attra-pub/nematode.html.

Nickle, W.R. (Ed.). 1984. Plant and insect nematodes. Marcel Dekker, New York. 925 pp.

Potter, J.W. et T.H.A. Olthof. 1974. Yield losses in fallmaturing vegetables relative to population densities of Pratylenchus penetrans and Meloidogyne hapla. Phytopathology 64 : 1072-1075.

Rousson, J. 2005. Liste des parasites réglementés par le Canada. Agence canadienne d'inspection des aliments, Direction des produits végétaux, Division de la protection des végétaux. http://www.inspection.gc.ca/francais/ plaveg/protect/listpesparf.shtml.

Société de protection des plantes du Québec (SPPQ). 2003. Noms des maladies des plantes au Canada. $4^{\mathrm{e}}$ éd. Société de protection des plantes du Québec, Québec. 340 pp. 